\title{
Corrigendum: MHC-dependent inhibition of uterine NK cells impedes fetal growth and decidual vascular remodeling
}

\author{
Jens Kieckbusch, Louise M. Gaynor, Ashley Moffett \& Francesco Colucci
}

Nature Communications 5:3359 doi: 10.1038/ncomms4359 (2014); Published 28 Feb 2014; Updated 20 Apr 2017

This Article was originally published under a CC BY-NC-ND 3.0 license, but has now been made available under a CC BY 4.0 license. The PDF and HTML versions of the paper have been modified accordingly. 